\title{
QUALIDADE DE MANGA 'TOMMY ATKINS' DA PRODUÇÃO INTEGRADA RECOBERTA COM FÉCULA DE MANDIOCA ASSOCIADA A ÓLEOS ESSENCIAIS E QUITOSANA ${ }^{1}$
}

\author{
LUAN PEDRO MELO AZERÊDO², SILVANDA DE MELO SILVA, \\ MARIA AUXILIADORA COELHO LIMA ${ }^{4}$, RENATO LIMA DANTAS 5 , \\ WALTER ESFRAIN PEREIRA ${ }^{3}$
}

RESUMO - Este trabalho teve por objetivo avaliar a qualidade de manga 'Tommy Atkins' da Produção Integrada (PI) recoberta com fécula de mandioca (F) associada a óleos essenciais e quitosana. A manga foi colhida na maturidade comercial de pomar certificado na PI. Os recobrimentos foram: Controle; F a 3\%; F a $3 \%$ + erva-doce a $0,9 \%$ (FED); F a 3\%+ orégano a $0,5 \%$ (FO), e F a $2 \%+$ quitosana a $2 \%(\mathrm{FQ})$. As mangas recobertas foram mantidas por 20 dias a $12 \pm 1^{\circ} \mathrm{C}$ e $80 \pm 2 \%$ U.R. e, em seguida, transferidas para a condição ambiente $\left(24 \pm 2{ }^{\circ} \mathrm{C} ; 75 \pm 4 \%\right.$ U.R) por mais 12 dias, simulando a condição de mercado. A combinação FQ retardou o amadurecimento, mantendo a coloração, os teores de sólidos solúveis e de acidez, apresentando mangas com intenção de compra e sabor superiores ao controle.

Termos para Indexação: Fluorescência da clorofila; Foeniculum vulgare, Origanum vulgare, recobrimentos comestíveis.

\section{QUALITY OF 'TOMMY ATKINS' MANGO FROM INTEGRATED PRODUCTION COATED WITH CASSAVA STARCH ASSOCIATED WITH ESSENTIAL OILS AND CHITOSAN}

\begin{abstract}
This study had as objective to evaluate the quality of 'Tommy Atkins' mango from the Integrated Production (IP) coated with cassava starch (S) associated with essential oils and chitosan. Mango was harvested, at the commercial maturity, from a certified orchard by the Mango IP. The coatings were: Control; S 3\%; S 3\% + Foeniculum 0.9\% (SF); S 3\% + Origanum $0.5 \%$ (SO), and S 2\% + Chitosan 2\% (SCH). Coated mangos were stored for 20 days at $12 \pm 1{ }^{\circ} \mathrm{C}$ and $80 \pm 2 \% \mathrm{RH}$ and, following, transferred to room conditions $\left(24 \pm 2{ }^{\circ} \mathrm{C} ; 75 \pm 4 \% \mathrm{RH}\right)$ for more 12 days, simulating the marketing condition. The combination $\mathrm{SCH}$ delayed ripening, maintaining the coloration, soluble solids, and acidity contents, providing mangos with purchase intention and flavor higher than the control.

Index Terms: Chlorophyl fluorescence; Foeniculum vulgare, Origanum vulgare, edible coatings.
\end{abstract}

${ }^{1}$ (Trabalho 270-14). Recebido em: 28-10-2014. Aceito para publicação em:14-08-2015.

${ }^{2}$ Mestrando/PPGCTA/UFPB, 58000-000,João Pessoa-PB. E-mail: luan_p22@hotmail.com

${ }^{3}$ Prof. PhD, CCA/UFPB,58397-000,Areia-PB. E-mails: silvasil@cca.ufpb.br (autor correspondente); wep@cca.ufpb.br

${ }^{4}$ Dra.Pesq. Embrapa Semiárido, BR 428, Km 152, 56.302-970, Petrolina-PE. E-mail: maclima@cpatsa.embrapa.br

${ }^{5}$ Doutorando/PPGA/CCA/UFPB, 58.397-000, Areia -PB. E-mail: renato_dantas@hotmail.com.br 


\section{INTRODUÇÃO}

O Brasil produziu 1,163 milhão de toneladas de mangas em 2013, destacando-se os Estados da Bahia, São Paulo e Pernambuco, que, juntos, foram responsáveis por $77,25 \%$ da produção brasileira (IBGE, 2013). Com esses resultados, o Brasil é o sétimo maior produtor e o quarto maior exportador de manga do mundo (FAO, 2015). A cultivar 'Tommy Atkins' responde por $80 \%$ dos plantios de manga no Brasil em função de sua maior resistência a pragas e doenças, e por apresentar maior conservação póscolheita (FONSECA et al., 2006).

O Sistema de Produção Integrada (PI) minimiza o uso de agroquímicos, preservando o meio ambiente, assim como a saúde do consumidor e do produtor, assegurando a obtenção de frutos com alta qualidade (MARTINS et al., 2012). Em 2008, 47\% da área total do Vale do Submédio São Francisco destinada à cultura da mangueira já havia aderido à PI (LOPES et al., 2009). A adesão à PI possibilita ao produtor a obtenção do selo "Brasil Agricultura de Qualidade" do MAPA, agregando valor à produção (GASPARINI, 2014).

A associação de recobrimentos orgânicos, a exemplo de fécula de mandioca, com óleos essencias, pode representar uma alternativa promissora na conservação pós-colheita da manga fresca (LIMA et al., 2012) e minimamente processada (ALIKHANI, 2014), incrementando o valor agregado de frutos da PI. O desenvolvimento de recobrimentos biodegradáveis/comestíveis baseado na combinação de biopolímeros tem atraído a atenção devido à sua abundância e ao potencial de substituição em relação a alguns produtos petroquímicos na embalagem de frutos e hortaliças (KALIA; PARSHAD, 2014). Neste sentido, a combinação de quitosana com óleo essencial de limão reduziu a perda de massa e a incidência de podridões em morango (PERDONES et al., 2012), em uvas 'Moscatel' recobertas com quitosana com óleo de bergamota (SÁNCHEZGONZÁLEZ et al., 2011) e em mamão recoberto com quitosana (GALO et al., 2014) .

Por isso, o objetivo deste trabalho foi avaliar a qualidade de mangas 'Tommy Atkins' recobertas com fécula de mandioca associada a óleos essenciais e quitosana.

\section{MATERIAL E MÉTODOS}

Manga (Mangifera indica L.) da cultivar Tommy Atkins foi colhida no período da manhã, na maturidade comercial (estádio II- 20\% de casca verde, $50 \%$ de vermelha e $30 \%$ de amarela) de empresa exportadora certificada no Sistema de Produção Integrada de Mangas, localizada em Petrolina - PE, adotando-se os procedimentos de Boas Práticas Agrícolas (LIMA et al., 2012), sendo acondicionadas em caixas plásticas e cada fruto revestido com papel-manteiga. As mangas foram transportadas para o Laboratório de Biologia e Tecnologia Pós-Colheita do CCA/UFPB, localizado em Areia-PB.

A quitosana foi adquirida da Polymar Ind. S/A (Fortaleza-CE, Brasil), com 95\% da granulometria de 80 mesh, grau de desacetilação de $85-90 \%$ e PM de $340 \mathrm{kDa}$. A fécula de mandioca (Manihot esculenta Crantz) foi preparada no laboratório, a partir de raízes da variedade Cedinha, extraída conforme Lima et al. (2012). Os óleos essenciais de erva-doce (Foeniculum vulgare Mill.) e orégano (Origanum vulgare L.) de grau alimentar foram adquiridos da Ferquima Ind. e Com. Ltd. (Vargem Grande PaulistaSP, Brasil).

A solução de fécula de mandioca foi preparada na concentração de 2 e 3\% (p/v) (LIMA et al., 2012) através de geleificação do amido, pelo aquecimento da solução até $70{ }^{\circ} \mathrm{C}$, sob constante agitação, sendo em seguida resfriada para o recobrimento dos frutos. A solução de quitosana foi preparada a $2 \%(\mathrm{p} / \mathrm{v})$ (ZHU et al., 2008), em solução de ácido acético glacial a $1 \%$, sob agitação por 2 horas, até completa homogeneização. Para a incorporação de óleos essenciais aos recobrimentos, foram utilizados 9 $\mathrm{mL}$ de óleo de erva-doce para cada litro $(0,9 \%)$ de solução de fécula de mandioca (TORRES, 2010) e 5 mL. $\mathrm{L}^{-1}$ de óleo de orégano $(0,5 \%)$ para a solução de fécula de mandioca (SANTOS et al., 2012). Os óleos essenciais foram adicionados de Tween $40^{\circledR}(0,1 \%$ $\left.\mathrm{mL} . \mathrm{L}^{-1}\right)$ e glicerol $\left(1,5 \% \mathrm{~mL} . \mathrm{L}^{-1}\right)$, sendo o primeiro para melhorar a homogeneização, e o segundo, para maximizar as propriedades de plastificação dos recobrimentos (SALVIA-TRUJILLO et al., 2015).

Os frutos foram sanificados com solução de hipoclorito de sódio a $100 \mathrm{mg} . \mathrm{L}^{-1}$, por 3 minutos. Após secagem sob condição ambiente, os recobrimentos aplicados aos frutos foram: controle (C), sem recobrimento; fécula de mandioca a 3\% (F); F a 3\% + óleo de erva-doce a 0,9\% (FED); F a 3\% + óleo de orégano a $0,5 \%$ (FO) e F a $2 \%$ + quitosana a $2 \%$ (FQ). Os frutos foram imersos por 30 segundos nas soluções dos respectivos recobrimentos e secos ao 
ar com auxílio de ventilador, sobre telas de aço inox. Após a secagem, os frutos foram acondicionados em bandejas de poliestireno expandido e armazenados a $12 \pm 1{ }^{\circ} \mathrm{C}$ e $80 \pm 2 \%$ U.R. por 20 dias e, posteriormente, transferidos para condição ambiente $\left(24 \pm 2{ }^{\circ} \mathrm{C}\right.$ e $75 \pm 4 \%$ U.R) por mais 12 dias, sendo avaliados a cada cinco dias no armazenamento refrigerado e a cada três dias na condição ambiente.

Avaliações: Perda de massa (\%), pelo percentual acumulado obtido por diferença em relação à massa inicial; Firmeza $(\mathrm{N})$, através do penetrômetro manual Magness Taylor Pressure Tester em duas leituras equidistantes; Cor de fundo, obtida com colorímetro Minolta, nos parâmetros: luminosidade $\left(\mathrm{L}^{*}\right)$, cromaticidade $\left(\mathrm{C}^{*}\right)$ e o ângulo Hue $\left({ }^{\circ} \mathrm{H}\right)$; Sólidos solúveis (SS), por duas leituras diretas com refratômetro tipo Abbe digital, ATAGO $\mathrm{N} 1$; Acidez titulável (AT $-\mathrm{g}$ ácido cítrico. $100 \mathrm{~g}^{-1} \mathrm{de}$ polpa), por titulometria com solução de $\mathrm{NaOH} 0,1 \mathrm{M}$ (MIGUEL et al., 2014); relação SS/AT, quociente de SS por AT; $\mathrm{pH}$, com potenciômetro e teor de açúcares redutores e não redutores, conforme (Lima et al.(2012).

A fluorescência das clorofilas foi medida com fluorímetro Pocket PEA, tomando quatro pontos equidistantes para a realização das leituras na casca da manga. Foram quantificados os parâmetros $F_{o}$ (fluorescência inicial), $F_{m}$ (fluorescência máxima) e a relação $F_{v} / F_{m}$ (LECHAUDEL et al., 2010).

A análise sensorial foi realizada em duas etapas, sendo analisados inicialmente os atributos de aparência e a caracterização do sabor da Amostra Controle-Referência (R) e, posteriormente, foi avaliado o sabor das amostras de frutos com cada recobrimento por meio do teste de comparação múltipla. O método empregado para a avaliação de atributos do sabor da $\mathrm{R}$ e da aparência foi a Análise Descritiva Quantitativa (ADQ), (QUEIROZ; TREPTOW, 2006). Doze provadores, previamente treinados, receberam pedaços de frutos de aproximadamente $1 \mathrm{~cm}$, acompanhados de uma ficha com escala não estruturada de $9 \mathrm{~cm}$, ancorada por termos descritivos, onde o julgador marcava com um traço vertical a intensidade da característica solicitada, referente ao sabor, textura e aparência. Para a avaliação do sabor, os provadores receberam duas amostras iguais por vez, de mangas dos quatro recobrimentos, acompanhadas da referência $(\mathrm{R})$, que correspondia ao controle codificado. Os atributos avaliados para sabor e textura foram: sabor doce, sabor ácido e suculência. Aos provadores solicitou-se que assinalassem em cada amostra se esta era igual ou diferente, e o grau de diferença de acordo com a escala de categoria, que variou de 1 - muitíssimo inferior a R, a 9 - muitíssimo superior a $\mathrm{R}$, sendo que 5 - era igual a R. Os provadores receberam as amostras codificadas com um número de três dígitos, acompanhado de água mineral para a limpeza do palato.

O delineamento foi o inteiramente casualizado, em esquema fatorial $5 \times 2$, sendo cinco recobrimentos e duas condições de armazenamento. A análise estatística considerou o aninhamento dos dias de armazenamento dentro de cada ambiente e a interação entre recobrimentos com os dois ambientes. Nas análises destrutivas, considerou-se o esquema fatorial, enquanto nas não destrutivas, o de parcelas subdivididas no tempo. Os resultados foram submetidos à ANOVA, o efeito dos dias de armazenamento foi avaliado mediante regressão polinomial, para ambientes e recobrimentos, e o teste de Tukey e Dunnett, a 5\% de probabilidade, para as sensoriais de aparência e sabor, respectivamente.

\section{RESULTADOS E DISCUSSÃO}

A perda de massa foi menor para mangas recobertas com a combinação fécula de mandioca e quitosana (FQ) no armazenamento refrigerado. A perda de massa aumentou bruscamente quando os frutos foram transferidos para a condição ambiente (Figura 1A), em decorrência do aumento da taxa metabólica ocasionada pelo aumento da temperatura. A $24{ }^{\circ} \mathrm{C}$, mangas recobertas com fécula, associada à erva-doce (FED), mostraram a menor perda de massa em relação aos demais recobrimentos, como reportado por Lima et al. (2012).

A firmeza da polpa foi mantida no armazenamento refrigerado, sobretudo para mangas do recobrimento $\mathrm{F}$ (Figura 1B). A transferência dos frutos para o ambiente resultou em perda acentuada, observando-se um declínio de cerca de $50 \%$ após 3 dias a $24{ }^{\circ} \mathrm{C}$ e, aos 12 dias, de cerca de $95 \%$ da firmeza. O amadurecimento de mangas é caracterizado por amaciamento da polpa e pela mudança de coloração (ZHU et al., 2008), indicando, neste experimento, que o amadurecimento evoluiu após a transferência.

Mangas de todos os recobrimentos acumularam sólidos solúveis (SS), sobretudo o FED (13,8\%), na refrigeração. Entretanto, frutos recobertos apenas com $\mathrm{F}$ apresentaram o menor acúmulo $(9,8 \%)$. Na transferência para a condição ambiente, os frutos mantiveram teores de SS próximos a $13 \%$ durante o armazenamento (Figura 2A). Entretanto, Cissé et al. (2015) reportaram SS superiores a $13 \%$ para mangas 'Kent' recobertas com quitosana, que não diferiu do controle. 
A acidez titulável (AT) diminuiu levemente no armazenamento a $12{ }^{\circ} \mathrm{C}$, porém quando as mangas foram transferidas para $24^{\circ} \mathrm{C}$, observou-se um brusco declínio na $\mathrm{AT}$, provavelmente pela utilização dos ácidos orgânicos no Ciclo de Krebs, na geração de energia e na gliconeogênese (LECHAUDEL et al., 2010). No entanto, em frutos recobertos com FQ, os teores de AT foram superiores (Figura 2B), possivelmente em decorrência da menor taxa metabólica. Mangas 'Kent' recobertas com quitosana e lactoperoxidase apresentaram redução da taxa respiratória e manutenção dos teores de ácidos (CISSÉ et al., 2015).

A relação $\mathrm{SS} / \mathrm{AT}$ manteve-se abaixo de 10 unidades, a $12{ }^{\circ} \mathrm{C}$, em mangas de todos os recobrimentos, aumentando após a transferência para o ambiente, principalmente para o controle (Figura 2C), em decorrência do amadurecimento. Mangas recobertas com fécula associada a orégano (FO), seguido de FQ, apresentaram as mais baixas relações SS/AT na condição ambiente, que pode ser decorrente do retardo no amadurecimento, como também reportado para manga 'Tainong' recoberta com quitosana, por Zhu et al. (2008).

Os teores de AR mantiveram-se estáveis, independentemente da condição de armazenamento (Figura 2D). Por sua vez, os teores de ANR aumentaram durante os 20 dias de refrigeração, em mangas recobertas com FED, tendo o maior teor $\left(4,27\right.$ g. $\left.100 \mathrm{~g}^{-1}\right)$ (Figura 2E), indicando que sacarose se acumula na pós-colheita. Na condição ambiente, os teores de ANR atingiram o valor máximo aos 26 dias para frutos dos recobrimentos F $(5,13$ g. 100 $\left.\mathrm{g}^{-1}\right)$ e FED $\left(5,18\right.$ g. $\left.100 \mathrm{~g}^{-1}\right)$. O comportamento dos açúcares totais foi similar aos ANRs (Figura 2F) e estão de acordo com Torres (2010), em manga 'Tommy Atkins' da PI.

Para a coloração da casca, os valores de $\mathrm{L}^{*}$ e os de $\mathrm{C}^{*}$ aumentaram na manga 'Tommy Atkins' em todos os recobrimentos, porém com menor intensidade na do FQ, indicando a marcante influência da quitosana em retardar a evolução da coloração (Figuras $3 \mathrm{~A}, \mathrm{~B}, \mathrm{C}$ ), como também reportado por Zhu et al. (2008), em mangas 'Tainong'. $\mathrm{O}$ valor de ${ }^{\circ} \mathrm{H}$ diminuiu durante o armazenamento, principalmente após a transferência da refrigeração para $24{ }^{\circ} \mathrm{C}$. Entretanto, o valor de ${ }^{\circ} \mathrm{H}$ em frutos com FQ manteve-se superior aos demais. Este efeito da quitosana, portanto, deve ter sido aditivo, já que o uso de fécula de mandioca, isoladamente, retardou o desenvolvimento da coloração desta manga (LIMA et al., 2012).

Os valores de $F_{o}$ declinaram em mangas sob refrigeração com todos os recobrimentos, enquanto na condição ambiente o controle mostrou menor valor de $F_{o}$, como resultado da perda de clorofila. Em contraste, mangas do FQ apresentaram aumento em $F_{o}$ ao final do armazenamento, refletido na maior retenção da clorofila. Em bananas tratadas com etileno e armazenadas a 20 e a $30^{\circ} \mathrm{C}$, por sete dias, $F_{o}$ diminuiu, principalmente a $20^{\circ} \mathrm{C}$ (YANG et al., 2011). Lechaudel et al. (2010), em estudo com mangas 'Cogshall' que amadureceram na planta, reportaram declínio de $F_{o}$, próximo ao controle (C) deste experimento (Figura 3D).

Os valores de $F_{m}$ na manga 'Tommy Atkins' diminuíram durante o armazenamento (Figura 3E). Essa redução foi maior em frutos recobertos com $\mathrm{F}$, FED, FO e C. Entretanto, os de FQ mantiveram-se com valores de $F_{m}$ mais elevados. A diminuição concomitante na $F_{o}^{m}$ e $F_{m}$ em mangas está relacionada com a diminuição da atividade dos cloroplastos associada à evolução do climatério da fruta (LECHAUDEL et al., 2010). Em manga 'Toinong', no entanto, a adição de quitosana ao recobrimento retardou em cerca de sete vezes a degradação da clorofila (ZHU et al., 2008), corroborando, neste trabalho, os maiores valores de $F_{m}$ para mangas recobertas com a combinação fécula-quitosana (FQ).

A fluorescência das clorofilas é uma ferramenta potente no monitoramento do status dos cloroplastos (LECHAUDEL et al., 2010), sendo $F_{v}$ $F_{m}$ frequentemente usado para ilustrar a atividade do fotosistema II (PSII) em frutos (YANG et al., 2011). A relação $F_{v} / F_{m}$ declinou levemente no armazenamento a $12{ }^{\circ} \mathrm{C}$, com acentuada queda a $24{ }^{\circ} \mathrm{C}$. No entanto, o FQ manteve os valores mais elevados, pelo retardo no amadurecimento. Isto também foi reportado por Lechaudel et al. (2010), em mangas 'Cogshall', que mantiveram os valores $F_{v} / F_{m}$ no início do armazenamento ao ambiente. Em bananas, $F_{v} / F_{m}$ foi 0,8 no início e declinou para 0,16 ao final do armazenamento, em decorrência do amadurecimento e do aumento da produção de etileno (YANG et al., 2011), inferior ao aqui reportando para FQ.

A cor de fundo da manga 'Tommy Atkins' (vermelho-alaranjada) aumentou durante o armazenamento, caracterizando como moderado em frutos recobertos com $\mathrm{F}, \mathrm{FO}$ e $\mathrm{C}$, enquanto aqueles com FED e FQ preservaram a coloração verde, sendo julgados como pouco vermelho-alaranjadas, como reportado por Lima et al. (2012). Manchas escuras surgiram nos de fécula de mandioca e óleos essenciais (FED e FO), sendo caracterizados entre regular e moderado. No caso do FED, a incidência de manchas foi inferior ao reportado por Lima et al. (2012), possivelmente em decorrência da maior dispersão do óleo na matriz do polímero. Por sua 
vez, no FQ, a incidência de manchas escuras foi insignificante. Não houve incidência de podridões em frutos de todos os recobrimentos até o $26^{\circ}$ dia, e apenas no FO, manteve-se como leve no $32^{\circ}$ dia. O recobrimento $\mathrm{FQ}$ obteve o valor máximo de intenção de compra no $26^{\circ}$ dia, enquanto o $\mathrm{C}$ se caracterizou entre moderada e muito moderada (Tabela 1 ).

Comparado ao Controle (C), quanto ao sabor doce, mangas recobertas com FED e FQ achavamse mais maduras no $20^{\circ}$ dia, diferindo do $\mathrm{C}$ por apresentarem maior valor de sabor doce, sendo, portanto, mais saborosas (Tabela 1). Entretanto, no $26^{\circ}$ dia, apenas mangas com $\mathrm{F}$ diferiram para menos, indicando estarem com maturação avançada. A manga com $\mathrm{F}$ apresentou maior sabor ácido no $20^{\circ}$ dia e também no $26^{\circ}$ dia, em conjunto com a FQ. Quanto à suculência, mangas com $\mathrm{F}$ e FED diferiram para menos, e as com FQ para mais em relação ao C, no $20^{\circ}$ dia de armazenamento, enquanto no $26^{\circ}$ dia mangas com $\mathrm{F}$ e FED continuaram com menor valor, e as com FO e FQ com maior valor de suculência.
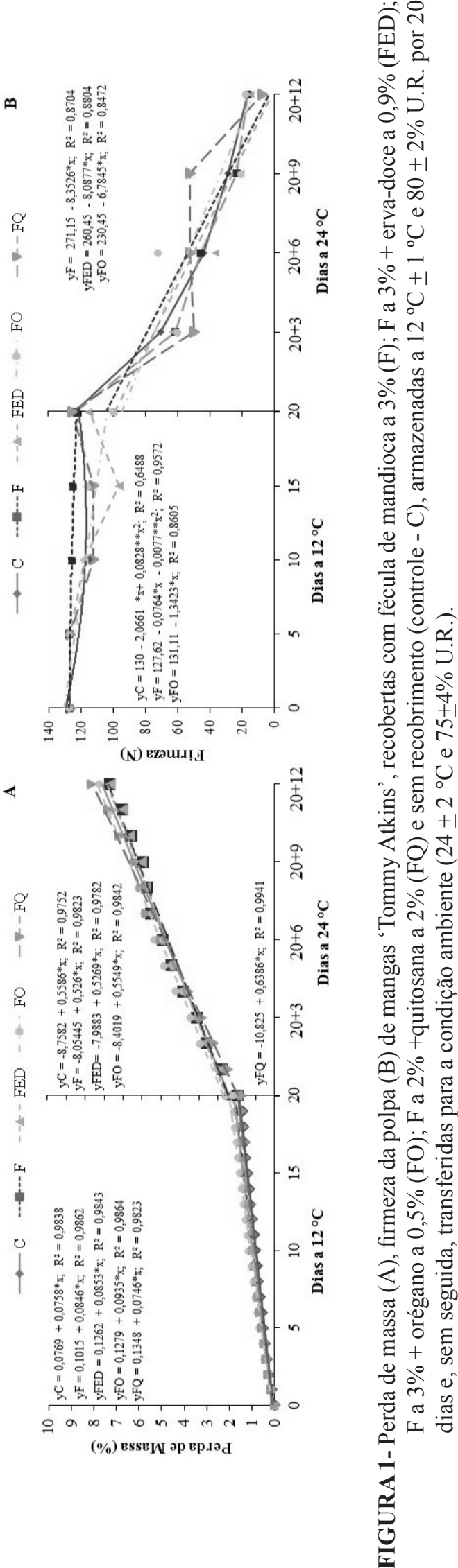


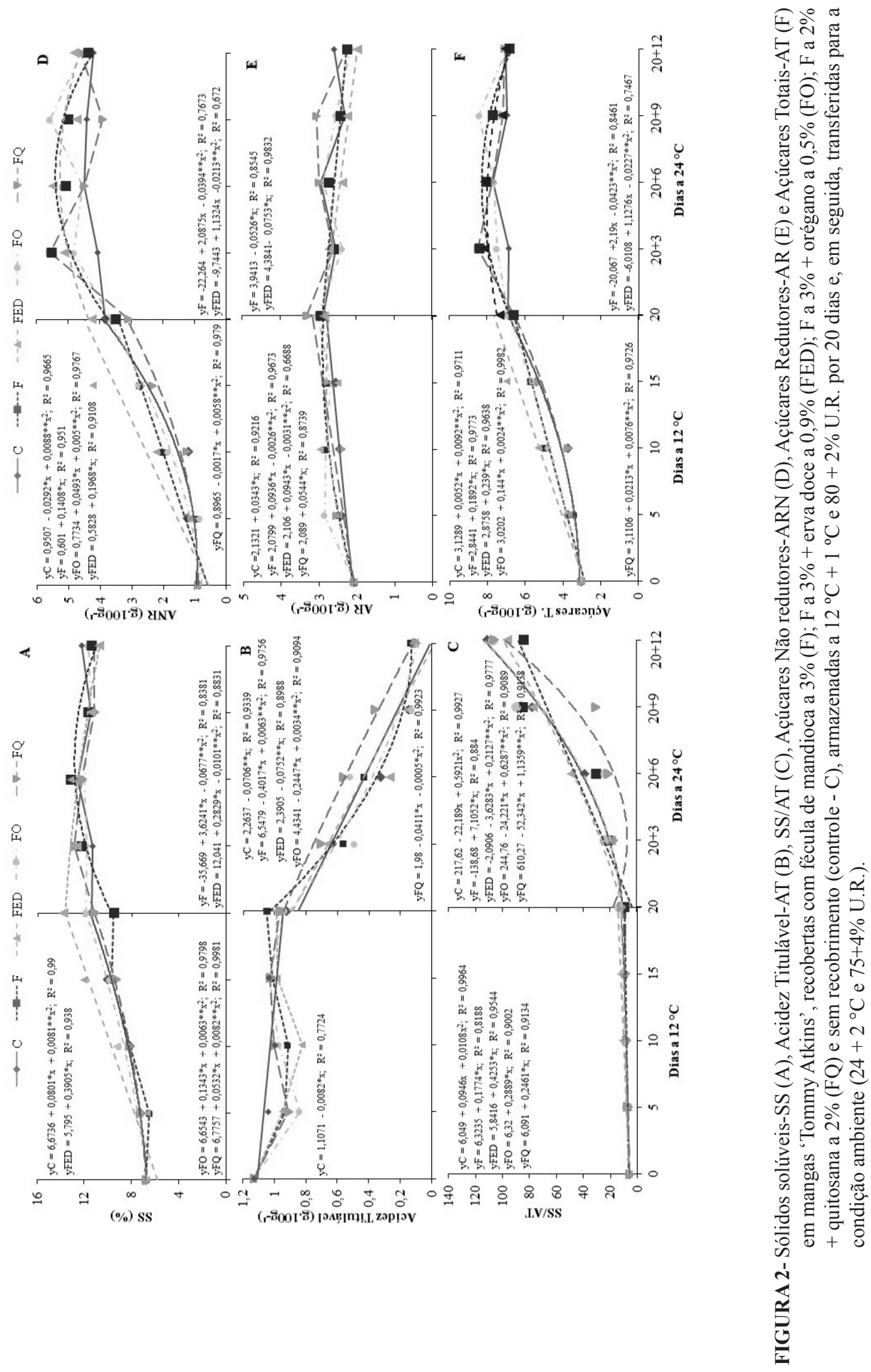



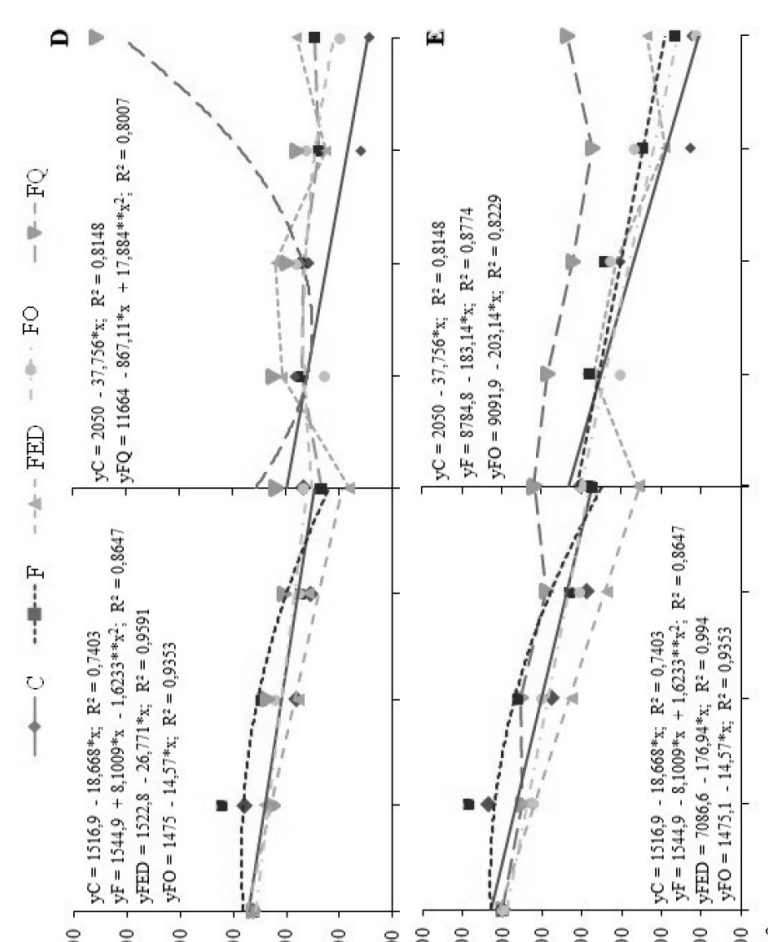

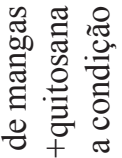

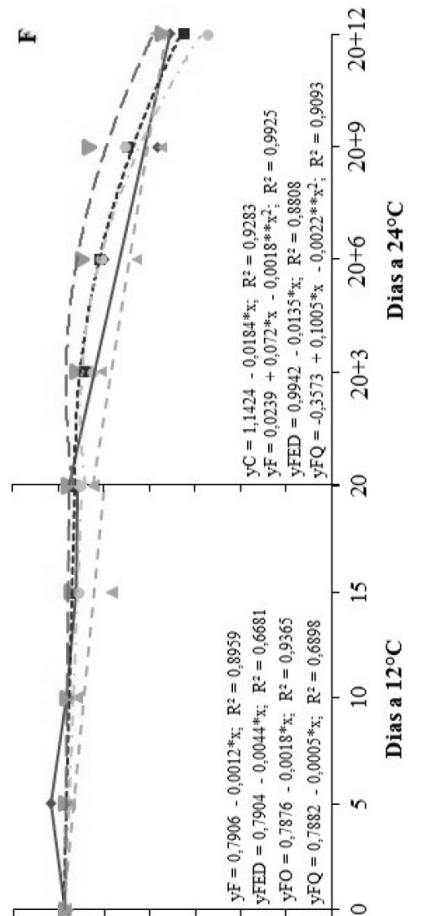

宅 $\stackrel{\circ}{\circ}$

छ ช

出: 垩

이

艩

욜 을

ป 00

(1)

\&

章䜦

घ + चี

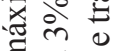

西

萡

बิํำ

近芒

후음

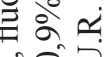

ใิ

0 ㅇํ

넌

.

永 +0

उ०

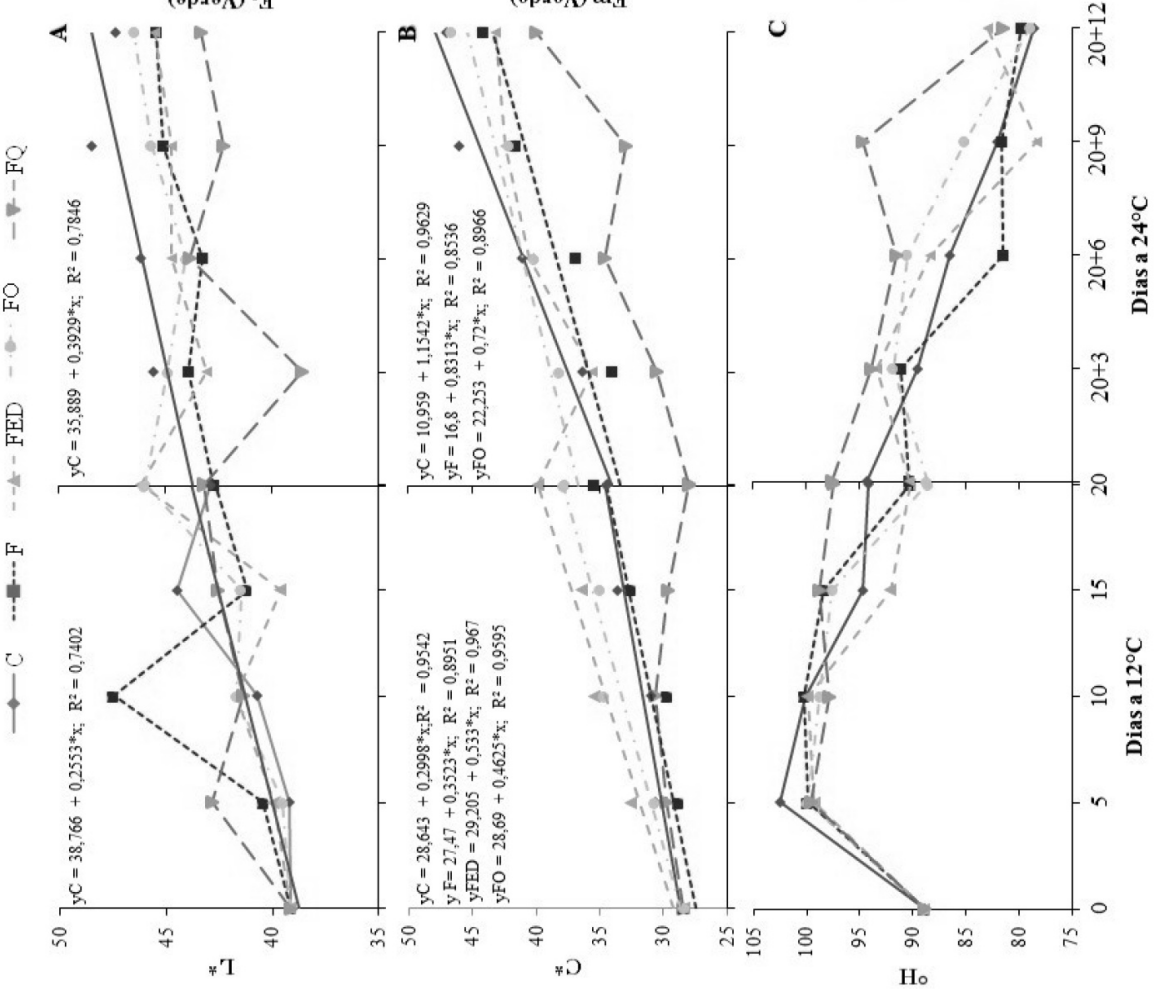

यू:

는

I

के ले है

ฮี ส

ह ช

:

응

कृ

U $\frac{\pi}{3} \frac{0}{0}$

胥苛

¿ छ

I

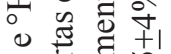

* 焉品

* 000

ํํำ

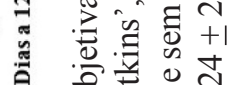

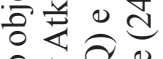

空远䒕

흠 ㅇํํ

ㅇํㅇ 윙 ส

它 
TABELA 1 - Atributos de aparência e de sabor da manga 'Tommy Atkins', recobertas com fécula a 3\% (F); fécula a $3 \%+$ erva doce a $0,9 \%$ (FED); fécula a $3 \%+$ orégano a $0,5 \%(\mathrm{FO})$; fécula a $2 \%+$ quitosana a $2 \%$ (FQ) e sem recobrimento (controle-C), armazenada a $12{ }^{\circ} \mathrm{C}+1{ }^{\circ} \mathrm{C}$ e $80 \pm 2 \%$ U.R. por 20 dias e transferidas por mais 12 dias para a condição ambiente $\left(24 \pm 2^{\circ} \mathrm{C}\right.$ e $75 \pm 4 \%$ U.R.).

\begin{tabular}{|c|c|c|c|c|c|c|}
\hline \multirow{2}{*}{ Atributos } & \multirow{2}{*}{ Dias } & \multicolumn{5}{|c|}{ Recobrimentos } \\
\hline & & $\mathrm{F}$ & FED & $\mathrm{FO}$ & FQ & $\mathrm{C}$ \\
\hline \multicolumn{7}{|c|}{ Aparência* } \\
\hline \multirow{5}{*}{ Manchas Escuras } & 0 & $0,0 \mathrm{a}$ & $0,0 \mathrm{a}$ & $0,0 \mathrm{a}$ & $0,0 \mathrm{a}$ & $0,0 \mathrm{a}$ \\
\hline & 5 & $0,1 b$ & $0,9 \mathrm{~b}$ & $5,4 \mathrm{a}$ & $0,1 \mathrm{~b}$ & $0,0 \mathrm{~b}$ \\
\hline & 20 & $5,5 \mathrm{a}$ & $6,3 \mathrm{a}$ & $6,5 \mathrm{a}$ & $0,2 \mathrm{~b}$ & $0,7 \mathrm{~b}$ \\
\hline & 26 & $7,2 \mathrm{a}$ & $2,5 b$ & $8,1 \mathrm{a}$ & $0,3 \mathrm{c}$ & $0,1 \mathrm{c}$ \\
\hline & 32 & $4,9 \mathrm{~b}$ & $6,2 \mathrm{ab}$ & $6,9 \mathrm{a}$ & $1,1 \mathrm{c}$ & $0,1 \mathrm{c}$ \\
\hline \multirow{5}{*}{ Podridão } & 0 & $0,0 \mathrm{a}$ & $0,0 \mathrm{a}$ & $0,0 \mathrm{a}$ & $0,0 \mathrm{a}$ & $0,0 \mathrm{a}$ \\
\hline & 5 & $0,0 \mathrm{a}$ & $0,0 \mathrm{a}$ & $0,0 \mathrm{a}$ & $0,0 \mathrm{a}$ & $0,0 \mathrm{a}$ \\
\hline & 20 & $0,2 \mathrm{a}$ & $0,2 \mathrm{a}$ & $0,1 \mathrm{a}$ & $0,1 \mathrm{a}$ & $0,1 \mathrm{a}$ \\
\hline & 26 & $1,8 \mathrm{a}$ & $2,1 \mathrm{a}$ & $0,1 b$ & $0,0 \mathrm{~b}$ & $0,1 b$ \\
\hline & 32 & $4,1 \mathrm{ab}$ & $2,9 b$ & $5,1 \mathrm{a}$ & $3,5 \mathrm{ab}$ & $4,1 \mathrm{ab}$ \\
\hline \multirow{5}{*}{ Intenção de Compra } & 0 & $7,0 \mathrm{a}$ & $7,0 \mathrm{a}$ & $7,0 \mathrm{a}$ & $7,0 \mathrm{a}$ & $7,0 \mathrm{a}$ \\
\hline & 5 & $5,2 \mathrm{ab}$ & $5,2 \mathrm{ab}$ & $3,8 b$ & $6,1 \mathrm{a}$ & $6,6 c$ \\
\hline & 20 & $2,1 \mathrm{c}$ & $1,2 \mathrm{c}$ & $1,9 \mathrm{c}$ & $6,4 \mathrm{~b}$ & $8,6 \mathrm{a}$ \\
\hline & 26 & $2,0 \mathrm{~b}$ & $1,3 b$ & $1,6 b$ & $9,0 \mathrm{a}$ & $7,8 \mathrm{a}$ \\
\hline & 32 & $0,3 \mathrm{c}$ & $1,2 \mathrm{c}$ & $1,1 \mathrm{c}$ & $5,3 \mathrm{a}$ & $3,5 b$ \\
\hline \multicolumn{7}{|c|}{ Sabor (Controle como Amostra Referência - R)** } \\
\hline \multirow{5}{*}{ Sabor doce } & 0 & 5,0 & 5,0 & 5,0 & 5,0 & 5,0 \\
\hline & 5 & 5,1 & 5,6 & 5,1 & 5,4 & 5,0 \\
\hline & 20 & 4,1 & $6,6^{*}$ & 4,4 & $7,3^{*}$ & 5,0 \\
\hline & 26 & $3,6^{*}$ & 4,6 & 5,3 & 5,4 & 5,0 \\
\hline & 32 & NA & NA & NA & NA & 5,0 \\
\hline \multirow{5}{*}{ Sabor ácido } & 0 & 5,0 & 5,0 & 5,0 & 5,0 & 5,0 \\
\hline & 5 & 5,1 & 5,3 & 4,9 & 5,0 & 5,0 \\
\hline & 20 & $6,7^{*}$ & 4,4 & 6,1 & 4,4 & 5,0 \\
\hline & 26 & $6,6^{*}$ & 5,1 & 5,7 & $6,4^{*}$ & 5,0 \\
\hline & 32 & NA & NA & NA & NA & 5,0 \\
\hline \multirow{5}{*}{ Suculência } & 0 & 5,0 & 5,0 & 5,0 & 5,0 & 5,0 \\
\hline & 5 & 5,0 & 5,0 & 5,1 & 5,6 & 5,0 \\
\hline & 20 & $3,7^{*}$ & $3,7^{*}$ & 4,3 & $7,0^{*}$ & 5,0 \\
\hline & 26 & $4,4^{*}$ & $4,4^{*}$ & $6,1^{*}$ & $5,9^{*}$ & 5,0 \\
\hline & 32 & NA & NA & NA & NA & 5,0 \\
\hline
\end{tabular}

*Médias seguidas pela mesma letra, nas linhas, não diferiram pelo teste de Tukey, a 5\% de probabilidade.

**Médias na horizontal diferem do Controle - referência (R) pelo teste de Dunnett a 5\%. As notas atribuídas variaram de 1 a 9 , sendo 1 extremamente menos que a $\mathrm{R}$ e 9 extremamente mais que a $\mathrm{R}$, e a nota 5 equivalente à amostra R. Escala não estruturada de $9 \mathrm{~cm}$. NA - Não Avaliado. 


\section{CONCLUSÃO}

Mangas recobertas com FED retiveram por mais tempo a coloração e apresentaram a menor incidência de podridões, entretanto observando-se manchas e menor suculência.

A combinação FQ manteve a atividade dos cloroplastos, retardou a mudança na coloração, manteve os valores de sólidos solúveis e acidez, apresentando maior intenção de compra e atributos de sabor superiores ao controle.

A manga 'Tommy Atkins' da PI, colhida na maturidade comercial, recoberta com fécula de mandioca associada a quitosana, apresentou maior retenção do amadurecimento e maior aceitação sensorial após a transferência da refrigeração para o ambiente, sendo, portanto, esta combinação uma promissora alternativa para a sua conservação póscolheita.

\section{AGRADECIMENTOS}

Ao BNB e CNPq, pelo apoio financeiro, e à CAPES, pela concessão da bolsa.

\section{REFERÊNCIAS}

ALIKHANI, M. Enhancing safety and shelf life of fresh-cut mango by application of edible coatings and microencapsulation technique. Food Science \& Nutrition, Dublin, v.2, n.3, p.210-217, 2014.

CISSÉ, M.; POLIDORI, J.; MONTE, D.; LOISEAU, G.; DUCAMP-COLLIN, M.N. Preservation of mango quality by using functional chitosanlactoperoxidase systems coatings. Postharvest Biology and Technology, Amsterdam, v.101, p.1014, 2015.

FAO. Food and Agriculture Organization of the United Nations. Roma: FAOSTAT Database Gateway-FAO. Disponível em: $\leq$ http://faostat3.fao. org/> Acesso em: 20 maio 2015.

FONSECA, N.; CUNHA, G.A.P.; NASCIMENTO, A.S.; SANTOS FILHO, H.P. A cultura da manga. 2.ed. Brasilia: Embrapa Informação Tecnologica, 2006. 73p.

GALO, J.Q.; SOUZA, M.L.; KUSDRA, J.F.; MATTIUZ, C.F.M. Conservação pós-colheita de mamão 'Sunrise Solo' com uso de quitosana. Revista Brasileira de Fruticultura, Jaboticabal, v.36, n.3, p.305-312, 2014.
GASPARINI, R. Produção integrada traz mais qualidade e segurança para frutas e verduras. 2014. Disponível em: $<$ http://www.agricultura.gov. br/comunicacao/noticias/2014/07/>. Acesso em: 28 maio 2015.

IBGE. Instituto Brasileiro de Geografia e Estatística. Produção agrícola estadual: lavoura permanente 2013. Disponível em: $\leq w w w . i b g e . g o v \cdot b r / e s t a d o s a t />$. Acesso em: Acesso em: 15 abr. 2015.

KALIA, A.; PARSHAD, V.R. Novel trends to revolutionize preservation and packaging of fruits/ fruit products: microbiological and nanotechnological perspectives. Critical Reviews in Food Science and Nutrition, London, v.55, n.2, p.159-82, 2014.

LECHAUDEL, M.; URBAN, L.; JOAS, J. Chlorophyll fluorescence, a nondestructive method to assess maturity of mango fruits (Cv. 'Cogshall') without growth conditions bias. Journal of Agricultural and Food Chemistry, Washington, v.58,n.13, p.75327538, 2010.

LIMA, A.B.; SILVA, S.M.; ROCHA, A.; NASCIMENTO, L.C.; RAMALHO, F.S. Conservação pós-colheita de manga 'Tommy Atkins' orgânica sob recobrimentos bio-orgânicos. Revista Brasileira de Fruticultura, Jaboticabal, v.34, n.3, p.704-710, 2012.

LOPES, P.R.C.; OLIVEIRA, J.E.M.; ASSIS, J.S.; SILVA, A.S.; BASTOS, D.C.; OLIVEIRA, I.V.M.; SILVA, R.R.S. Produção integrada de mangas. In: ZAMBOLIM, L.; NASSER, L.C.B.; ANDRIGUETO, J.R.; TEIXEIRA, J.M.; KOSOSKI, A.R.; FACHINELLO, J.C. Produção integrada no Brasil: agropecuária sustentavel, alimentos seguros. Brasília: Mapa/ACS, 2008. p.629 - 663.

MARTINS, L.P.; SILVA, S.M.; SILVA, A.P.; CUNHA, G.A.P.; MENDONÇA,R.M.N.; VILAR, L.C.; MASCENA, J.; LACERDA, J.T. Conservação pós-colheita de abacaxi 'Pérola' produzido em sistemas convencional e integrado. Revista Brasileira de Fruticultura, Jaboticabal, v.34, n.3, p.695-703, 2012.

MIGUEL, A.C.A.; DURIGAN, J.F.; BARBOSA, J.C.; MORGADO, C.M.A. Qualidade de mangas cv. Palmer após armazenamento sob baixas temperaturas. Revista Brasileira de Fruticultura, Jaboticabal, v.35, n.2, p.398-408, 2013. 
PERDONES, A.; SÁNCHEZ-GONZÁLEZ, L.; CHIRALT, A.; VARGAS, M. Effect of chitosanlemon essential oil coatings on storage-keeping quality of strawberry. Postharvest Biology and Technology, Amsterdam, v.70, p.32-41, 2012.

QUEIROZ, M.I.; TREPTOW, R.O. Análise sensorial para a avaliação da qualidade dos alimentos. Rio Grande: Ed. FURG, 2006. 268p.

SANTOS, N.S.T.; AGUIAR, A.J.A.A.; OLIVEIRA, C.E.V.; SALES, C.V.; SILVA, S.M.; Silva, R.S.; STAMFORD, T.C.M.; SOUZA, E.L. Efficacy of the application of a coating composed of chitosan and Origanum vulgare L. essential oil to control Rhizopus stolonifer and Aspergillus niger in grapes (Vitis labrusca L.). Food Microbiology, Amsterdam, v.32, n.2, p.345-53, 2012.

SÁNCHEZ-GONZÁLEZ, L.; PASTOR, C.; VARGAS, M.; CHIRALT, A.; GONZÁLEZMARTÍNEZ, C.; CHÁFER, M. Effect of hydroxypropylmethylcellulose and chitosan coatings with and without bergamot essential oil on quality and safety of cold-stored grapes. Postharvest Biology and Technology, Amsterdam, v.60, n.1, p.57-63, 2011.
SALVIA-TRUJILLO, L.; ROJAS-GRAÜ, M.A.; SOLIVA-FORTUNY, R.; MARTÍN-BELLOSO, $O$. Use of antimicrobial nanoemulsions as edible coatings: Impact on safety and quality attributes of fresh-cut Fuji apples. Postharvest Biology and Technology, Amsterdam, v.105, p.8-16, 2015.

TORRES, L.B.V. Qualidade e conservação póscolheita de mangas oriundas de sistemas de produção orgânica ou integrada. 2010. 239 f. Tese de Doutorado em Agronomia. Centro de Ciências Agrárias, Universidade Federal da Paraíba, 2010.

YANG, X.; SONG J.; FILLMORE, S.; PANG, X.; ZHANG, Z. Effect of high temperature on color, chlorophyll fluorescence and volatile biosynthesis in green-ripe banana fruit. Postharvest Biology and Technology, Amsterdam, v.62, n.3, p.246-257, 2011.

ZHU, X.; WANG, Q.; CAO, J.; JIANG, W. Effects of chitosan coating on postharvest quality of mango (Mangifera indica L. cv. 'Tainong') fruits. Journal of Food Processing and Preservation, Malden, v.32, n.5, p.770-784, 2008. 